\title{
Development of Hydroxyl Tagging Velocimetry for Low Velocity Flows
}

\author{
Matthieu A. André* and Philippe M. Bardet ${ }^{\dagger}$ \\ The George Washington University, Washington, DC, 20052 \\ Ross A. Burns ${ }^{\star}$ and Paul M. Danehy ${ }^{\S}$ \\ NASA Langley Research Center, Hampton, VA, 23681
}

\begin{abstract}
Hydroxyl tagging velocimetry (HTV) is a molecular tagging technique that relies on the photo-dissociation of water vapor into $\mathrm{OH}$ radicals and their subsequent tracking using laser induced fluorescence. Velocities are then obtained from time-of-flight calculations. At ambient temperature in air, the $\mathrm{OH}$ species lifetime is relatively short $(<50 \mu \mathrm{s})$, making it suited for high speed flows. Lifetime and radicals formation increases with temperature, which allows HTV to also probe low-velocity, high-temperature flows or reacting flows such as flames. The present work aims at extending the domain of applicability of HTV, particularly towards low-speed $(<10 \mathrm{~m} / \mathrm{s})$ and moderate $(<500 \mathrm{~K})$ temperature flows. Results are compared to particle image velocimetry (PIV) measurements recorded in identical conditions. Single shot and averaged velocity profiles are obtained in an air jet at room temperature. By modestly raising the temperature $\left(100-200{ }^{\circ} \mathrm{C}\right)$ the $\mathrm{OH}$ production increases, resulting in an improvement of the signal-to-noise ratio (SNR). Use of nitrogen - a non-reactive gas with minimal collisional quenching - extends the $\mathrm{OH}$ species lifetime (to over $500 \mu \mathrm{s})$, which allows probing of slower flows or, alternately, increases the measurement precision at the expense of spatial resolution. Instantaneous velocity profiles are resolved in a $100^{\circ} \mathrm{C}$ nitrogen jet (maximum jet-center velocity of $6.5 \mathrm{~m} / \mathrm{s}$ ) with an uncertainty down to $0.10 \mathrm{~m} / \mathrm{s}(1.5 \%)$ at $68 \%$ confidence level. MTV measurements are compared with particle image velocimetry and show agreement within $2 \%$.
\end{abstract}

\section{Nomenclature}

$\begin{array}{ll}T & =\text { temperature } \\ U & =\text { bulk velocity of the jet } \\ S N R & =\text { signal-to-noise ratio } \\ t_{0} & =\text { delay between write pulse and first read pulse } \\ \Delta t & =\text { time between first and second read pulses } \\ x & =\text { jet radial dimension }\end{array}$

\section{Introduction}

$\mathrm{M}$ olecular tagging velocimetry (MTV) [1] is an alternative to the well-established, non-intrusive flow diagnostics that is particle image velocimetry (PIV) [2]. Though MTV relies on a similar time-of-flight measurement technique to obtain velocity fields, the tracers are molecular species rather than discrete particles. MTV has been used in both gas- and liquid-phase fluids. In the gas phase, several techniques exist to "tag," or mark, the molecules of interest. The most common measurement scheme involves the creation or excitation of a radical chemical species and subsequently tracking its motion with planar laser-induced fluorescence (PLIF).

\footnotetext{
${ }^{*}$ Postdoctoral Scholar, Mechanical and Aerospace Engineering, 800 22 ${ }^{\text {nd }}$ St, NW, Washington DC 20052.

+ Assistant Professor, Mechanical and Aerospace Engineering, 800 22 nd St, NW, Washington DC 20052.

* Research Engineer, National Institute of Aerospace, AIAA Member.

$\S$ Research Scientist, Associate Fellow AIAA.
} 
Molecular tracers have the advantage of a response time and a settling velocity both equal to zero, which allows them to accurately follow quiescent and hypersonic flows alike [3]. Furthermore, these tracers can also be created in gas flows that are typically challenging to seed for PIV, such as high temperature or reacting flows, and in environment where particles are undesirable. The present technique is being developed for implementation in a high-temperature gas-cooled reactor, to measure coolant flow reversal for instance [4]. In this long-duration (several hours) flow, velocities are expected to be only a few meters/second, so if PIV were used, particle settling would be a concern. In this application, MTV is a viable alternative measurement technique. Some of the drawbacks of MTV include lower spatial resolution due to diffusion of tracers, limited tracer lifetime, and a more complicated setup compared to PIV. For instance, low speed flows $(\sim 2 \mathrm{~m} / \mathrm{s})$ have been successfully probed using ozone tagging velocimetry (OTV); however these tracers are not suited for high temperature due to reduced lifetime and $\mathrm{O}_{3}$ peak concentration [5].

Hydroxyl tagging velocimetry (HTV) makes use of hydroxyl radicals $(\mathrm{OH})$ created from water vapor. Advantages of this scheme are non-toxicity, ease of adding the tracer (water vapor) to the test gas, and relatively long tracer lifetime at high temperature [6]. $\mathrm{H}_{2} \mathrm{O}$ is also present at high temperature in most combustion product, which makes HTV a very convenient technique for probing such flows [7]. $\mathrm{OH}$ radicals are typically created with an Argon-Fluoride Excimer laser $(193 \mathrm{~nm})$ through a photo-dissociation process whose efficiency is temperature dependent. The dissociation fraction increases by an order of magnitude between ambient temperature and flame temperature [7]. OH offers several vibrational transitions in the ultraviolet (UV) portion of the spectrum, all from the $\mathrm{A}^{2} \Sigma^{+}-\mathrm{X}^{2} \Pi$ electronic transition, as shown in Fig. 1, that can be excited with PLIF. It should be noted that when exciting the $(0,0)$ band around $308 \mathrm{~nm}$, the resulting fluorescence occurs in the same spectral region and prevents the use of a filter to remove scattered laser light. Therefore, although the fluorescence signal is stronger in that case, the images are also more likely to be contaminated by scattering from particles and nearby surfaces. Thus, the SNR can be higher for $(1,0)$ excitation though the signal is lower.

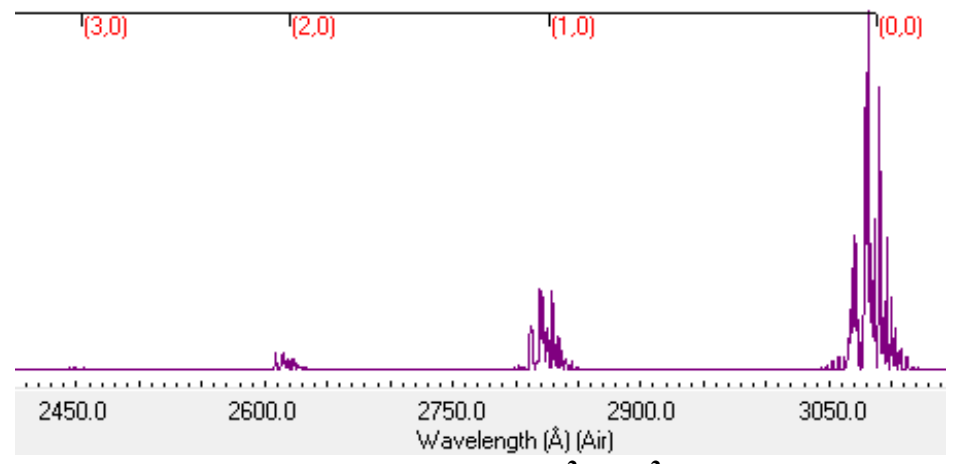

Figure 1: LIFBASE [8] computed absorption spectrum of $A^{2} \Sigma^{+}-X^{2} \Pi$ electronic transition of $\mathrm{OH}$ in air at 300 $K$ and $1 \mathrm{~atm}$. The first four vibrational transitions all originating from the ground vibrational state are shown here.

Table 1 lists a selection of HTV measurements with the main parameters of each study. This table confirms that the technique has been successfully applied for very-high-temperature flows or high-speed flows. In particular, the maximum reported $\Delta t$ is $30 \mu \mathrm{s}$ and $50 \mu \mathrm{s}$ at room temperature and at high temperature (>1400K), respectively. Note that these studies made use of a single read pulse, the initial tracer location being recorded in a separate step.

\begin{tabular}{|c|c|c|c|c|c|c|c|c|}
\hline Authors & Gas & $\mathrm{OH}$ band & $T(\mathrm{~K})$ & $\Delta t(\mu \mathrm{s})$ & $U(\mathrm{~m} / \mathrm{s})$ & SNR & Uncertainty & Uncertainty \\
\hline $\begin{array}{l}\text { Ribarov et al. } \\
{[6]}\end{array}$ & Air & $(0,0)(308 \mathrm{~nm})$ & 297 & 30 & 35.3 & 8 & $4 \mathrm{~m} / \mathrm{s}$ & $12 \%$ \\
\hline $\begin{array}{l}\text { Ribarov et al. } \\
\text { [7] }\end{array}$ & $\begin{array}{c}\text { Air } \\
\mathrm{H}_{2} \text {-air flame }\end{array}$ & $\begin{array}{l}(0,0)(308 \mathrm{~nm}) \\
(0,0)(308 \mathrm{~nm})\end{array}$ & $\begin{array}{c}300 \\
1400\end{array}$ & $\begin{array}{l}20 \\
50\end{array}$ & $\begin{array}{l}52 \\
10\end{array}$ & $\begin{array}{l}6 \text { to } 17 \\
11 \text { to } 53\end{array}$ & $\begin{array}{l}\text { N/A } \\
\text { N/A }\end{array}$ & $\begin{array}{l}\text { N/A } \\
\text { N/A }\end{array}$ \\
\hline Pitz et al. [5] & $\mathrm{H}_{2}$-air flame & $(3,0)(248 \mathrm{~nm})$ & 1450 & 20 & N/A & N/A & N/A & N/A \\
\hline Pitz et al. [9] & Air & $(1,0)(282 \mathrm{~nm})$ & 290 & 2 & 680 & 7 to 13 & $11 \mathrm{~m} / \mathrm{s}$ & $1.6 \%$ \\
\hline $\begin{array}{l}\text { Wehrmeyer, et } \\
\text { al. [10] }\end{array}$ & $\begin{array}{l}\mathrm{H}_{2} / \mathrm{N}_{2} \text {-air } \\
\text { flame }\end{array}$ & $(3,0)(248 \mathrm{~nm})$ & 2340 & 50 & 22 & N/A & $3 \mathrm{~m} / \mathrm{s}$ & $14 \%$ \\
\hline Lahr et al. [11] & Air & $(1,0)(282 \mathrm{~nm})$ & 290 & 2 & 730 & 5 to 11 & $8 \mathrm{~m} / \mathrm{s}$ & $1.1 \%$ \\
\hline
\end{tabular}

Table 1. Results from selected HTV studies. Uncertainty are given at $68 \%$ confidence level 
The present work explores the possibility of extending the use of HTV for measuring low velocity flows with the goal of sub-1-m/s, single-shot measurement uncertainty. Furthermore the current work extends the parametric domain over which HTV can be applied. Tests include temperature ranging from $300 \mathrm{~K}$ to $473 \mathrm{~K}$ (filling the gap between ambient and flame temperatures), excitation of the $(0,0)$ and $(1,0)$ bands, and air and $\mathrm{N}_{2}$ gas. Low-speed jets $(<10 \mathrm{~m} / \mathrm{s})$ are successfully probed with a $\Delta t$ of several hundred microseconds. HTV results are then compared with PIV results for the same flow, which is another novel aspect of this work. Measurement uncertainties are also assessed. A final aspect of the current work that improves on prior HTV work is the use of a two-probe laser system, instead of a single probe laser used in prior work. The two probe lasers allow truly single-shot measurement, providing both a reference and signal measurements for every excitation laser pulse. This approach defends against errors that could be caused by instantaneous (e.g. vibrations) or long term (e.g. thermal) misalignments. While this method has been used previously by Bathel et al [12] for $\mathrm{NO}_{2} \mathrm{MTV}$, we believe this is the first time it has been used for HTV.

\section{Instrumentation and Test Procedure}

\section{A. HTV Diagnostics}

The present HTV system is composed of an excimer laser (write pulse), a dual-pulse tunable dye laser (read pulses) and an intensified CCD camera (imager). The excimer laser, dye laser, and the two pump lasers are mounted on a cart to permit easy transport to experimental facilities. A diagram of this laser system is shown in Fig. 2.
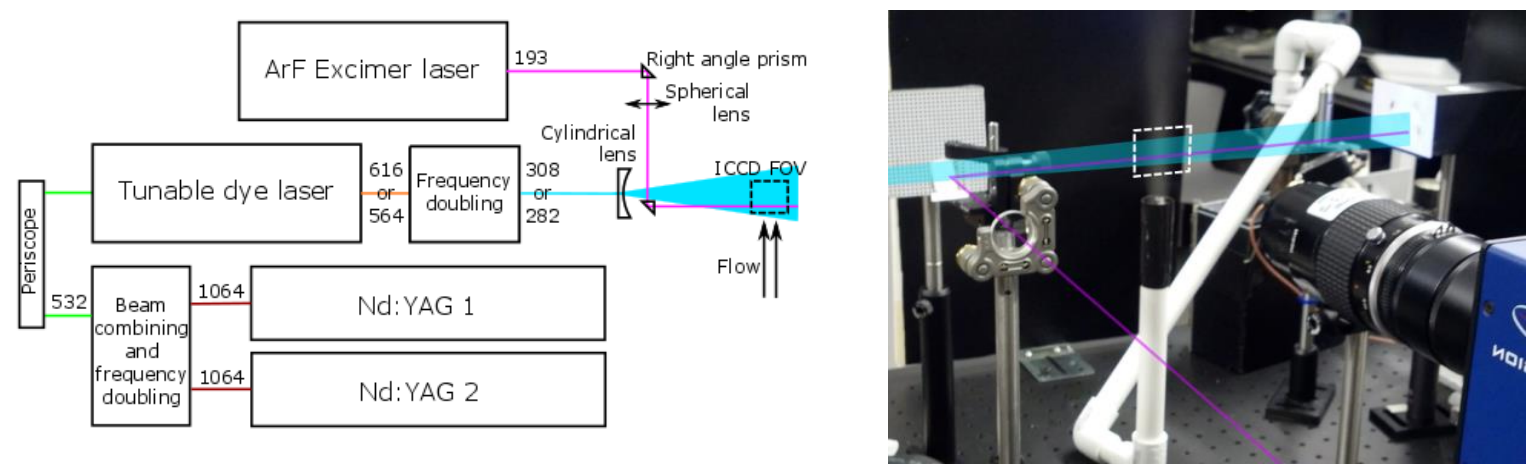

Figure 2: Left: Setup of the lasers used for "write" and "read" pulses. Wavelengths are given in nm. ICCD FOV stands for Intensified CCD field of view. Right: Photograph of the test section, with the FOV and laser beams highlighted.

The excimer outputs a $12 \mathrm{~mJ} /$ pulse beam at $193 \mathrm{~nm}$ to photo-dissociate $\mathrm{H}_{2} \mathrm{O}$ into $\mathrm{OH}$. The output of two frequency-doubled Nd:YAG lasers $(10 \mathrm{~Hz}, 0.5 \mathrm{~J} /$ pulse, $532 \mathrm{~nm}$ ) are combined to pump a tunable dye laser. This dual-pulse configuration allows correcting for potential beam wandering of the write laser, and hence leads to higher-accuracy measurements in comparison to using only a single read pulse. Using rhodamine dyes and a frequency-doubling BBO crystal, $\mathrm{A}^{2} \Sigma^{+}-\mathrm{X}^{2} \Pi$ transitions of the $\mathrm{OH}$ radical shown in Fig. 1 can be excited. The present work focuses on exciting the $(0,0)$ and $(1,0)$ vibrational transitions, around 308 and $282 \mathrm{~nm}$, respectively.

Fine tuning of the PLIF excitation wavelength maximizes the fluorescence signal. The $\mathrm{Q}_{1}(3)$ rotational transition in the $(0,0)$ band at $308.154 \mathrm{~nm}$ is used for resonant fluorescence. In this setup, fluorescence emission is around the same wavelength as the excitation, which prevents the use of spectral filters to remove scattered laser light. Using the $\mathrm{Q}_{1}(1)$ absorption line in the $(1,0)$ band at $281.905 \mathrm{~nm}$ allows using long-pass filters (Schott WG 295 and WG 305) to increase the SNR of the PLIF signal. However, the absorption at the $(1,0)$ transition is lower compared to the $(0,0)$ transition, as shown in Fig. 1, which results in a comparatively weaker signal for a given read pulse energy.

The fluorescence signal from the read pulses is recorded with a time-gated image intensifier (LaVision IRO25) coupled to a 12-bit CCD camera (QImaging QIClick). A UV-transmitting Nikon 105mm f/4.5 lens is mounted on the image intensifier with a $25.4 \mathrm{~mm}$ extension tube and provides a resolution of 21.4 pixels $/ \mathrm{mm}$. The gate time of the intensifier is set at $100 \mathrm{~ns}$ and temporally centered on the PLIF signal to suppress the background noise. This gate duration was chosen because it was expected to be much longer than the $\mathrm{OH}$ fluorescence lifetime, estimated to 
be a few nanoseconds. The CCD camera is connected to a workstation through a Firewire interface which allows images to be written directly to hard drive, enabling the recording of an extended duration of dataset (10 hours at 20 $\mathrm{Hz}$ ). Instruments are synchronized using a pulse generator (Berkeley Nucleonics 575) with an accuracy of $250 \mathrm{ps}$ and are monitored with a high-speed digital oscilloscope (Agilent MSOX-3054A).

\section{B. Test flow}

The HTV technique is first tested at ambient pressure and temperature. Compressed gas is pressure-regulated, bubbled through water in a TSI 6-jet atomizer, and exits a $15.8 \mathrm{~mm}$ inner diameter vertical pipe, shown in Fig. 2, right, forming a round jet in which velocity is to be measured. This setup is used for the $308 \mathrm{~nm}$ read pulse measurements.

In order to perform measurements on a heated jet, the tubing was switched from PVC to copper (7.9 mm inner diameter) and heat tape was wrapped around it. A thermocouple affixed to the tube exit provides feedback to regulate the temperature set point. This setup was used for the $282 \mathrm{~nm}$ read pulse measurements. The atomizer also enables seeding the jet with water droplets to serve as seeds for the PIV measurements. The mass flow rate was held constant for the various runs.

\section{Data acquisition and processing}

The excimer beam is focused with a $300 \mathrm{~mm}$ focal length spherical lens to a line perpendicular to the jet centerline. The dye laser beam is shaped into a vertical sheet that contains the path of the excimer beam, see the right side of Fig. 2. At $t=0 \mathrm{~s}$, the excimer laser is pulsed to create a horizontal line of $\mathrm{OH}$ radicals a few millimeters downstream of the nozzle exit. The tracers are then convected by the flow and their position is read a first time after a short delay, $t_{0}$, using a dye laser pulse. Their position is then read a second time, at $t=t_{0}+\Delta t$ with a second dye laser pulse. The image intensifier amplifies the signal to a level well above the read noise of the CCD camera. For each of the flow parameters described in Tab. 2, several hundred images are recorded. Images are processed with an in-house Matlab ${ }^{\circledR}$ code that performs cross correlation of the PLIF signal (binned in the horizontal direction to reduce noise) to measure the displacement. Sub-pixel accuracy is obtained by curve-fitting the correlation peak. Outliers are then rejected based on the difference from neighboring points. Mean velocity profiles and standard deviations are then computed.

\begin{tabular}{|l|c|c|c|c|}
\hline Run & Gas & OH band & $T(\mathrm{~K})$ & $\Delta t(\mu \mathrm{s})$ \\
\hline A & Air & $(0,0)(308 \mathrm{~nm})$ & 298 & 100 \\
\hline B & Air & $(0,0)(308 \mathrm{~nm})$ & 298 & 200 \\
\hline C & Air & $(1,0)(282 \mathrm{~nm})$ & 373 & 250 \\
\hline D & Air & $(1,0)(282 \mathrm{~nm})$ & 473 & 250 \\
\hline E & $\mathrm{N}_{2}$ & $(1,0)(282 \mathrm{~nm})$ & 373 & 250 \\
\hline F & $\mathrm{N}_{2}$ & $(1,0)(282 \mathrm{~nm})$ & 373 & 500 \\
\hline
\end{tabular}

Table 2: Summary of the HTV parameters of the present study

\section{Results}

\section{A. HTV at $308 \mathrm{nm:}(0,0)$ transition}

Raw instantaneous images recorded at several times after creation of the $\mathrm{OH}$ radicals are shown in Fig. 3. Although the dye laser can deliver up to $30 \mathrm{~mJ} /$ pulse, read pulse energy was kept around $4 \mathrm{~mJ}$ in each run to avoid sensor saturation by scattered light. In the top frame, the PLIF signal is strong and very close to a straight line, indicative of the short time delay between the write and the read pulse $\left(t=t_{0}=10 \mu \mathrm{s}\right)$. As the probe time was increased, the line of fluorescence convected visibly, with the largest displacements occurring at the jet centerline where the velocity was expected to be highest. Table 3 shows that there is a distinct trend of decreasing SNR, as $\Delta t$ was increased due to the diffusion and recombination of $\mathrm{OH}$ radicals with ambient species. 
The $\mathrm{OH}$ fluorescence is considerably stronger near the core of the jet, caused both by the increased concentration of water vapor and the focusing of the excimer laser beam through this region (thus producing higher concentrations of hydroxyl). The consequence of these effects is a further reduction in SNR near the edges of the jet and surrounding fluid. Finally, because the signal cannot be spectrally filtered, scattered light from dust particles in ambient air contaminates the images and further decreases the SNR.
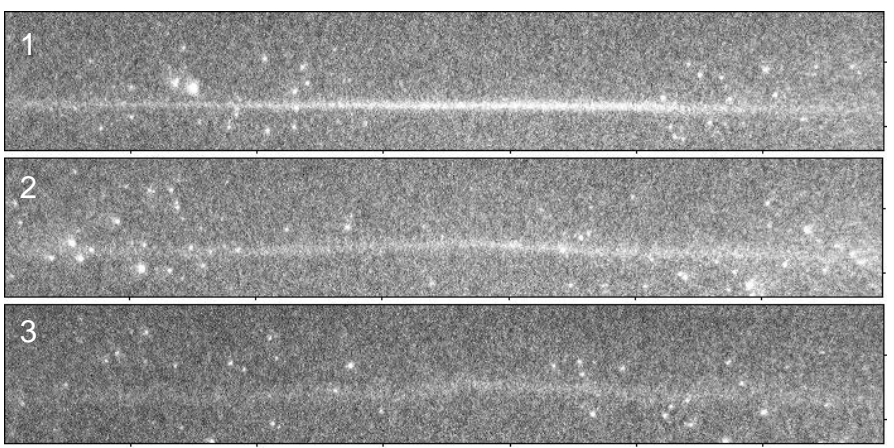

Figure 3: Raw instantaneous images recorded $10 \mu \mathrm{s}, 110 \mu \mathrm{s}$, and $210 \mu \mathrm{s}$ after radical formation (top, middle, and bottom, respectively)
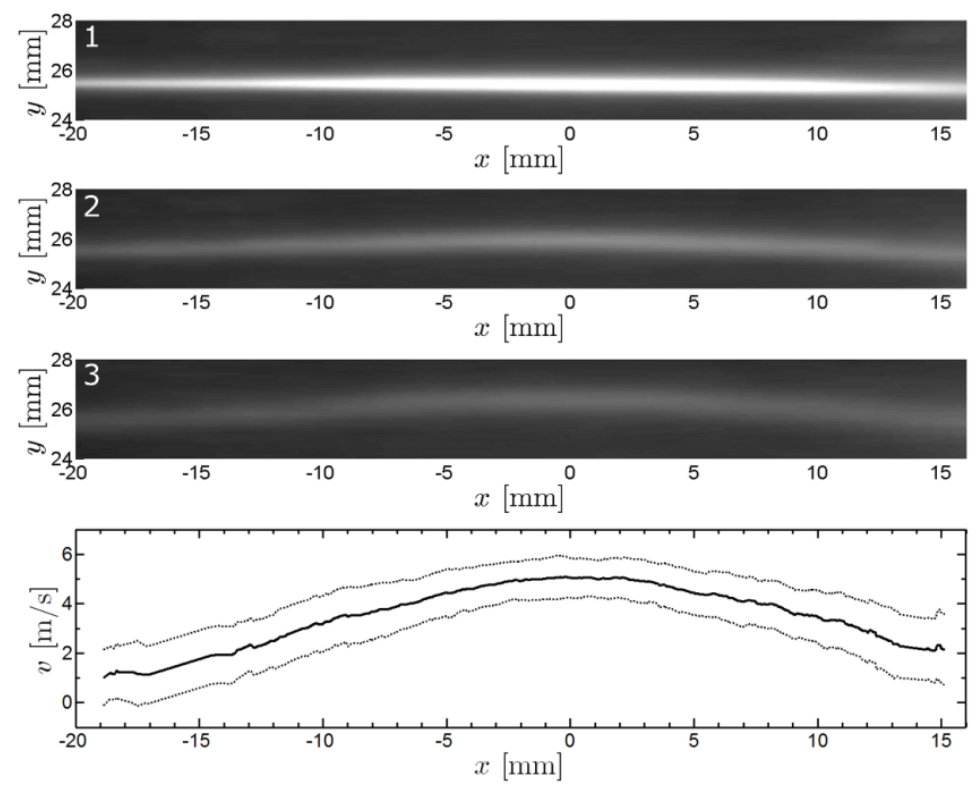

Figure 4: Mean OH-PLIF images resulting from the raw data shown in Fig. 3. Each image is obtained by averaging a minimum of $\mathbf{1 0 0}$ raw images. The velocity profile is computed using a custom curve-fitting algorithm between images sets 1 and 2 . The dotted lines indicate one standard deviation of the measured single-shot velocity distribution.

The results of the velocity calculations are shown in the final pane of Fig. 4. Due to the low SNR in the instantaneous images, column binning was performed over a region 64 pixels $(\sim 3 \mathrm{~mm})$ wide. It was found that the inclusive velocity range for the velocity profile here is between 1 and $5 \mathrm{~m} / \mathrm{s}$. The technique is thus capable of resolving velocities at this order of magnitude for ambient air.

\begin{tabular}{|l|c|c|c|c|}
\cline { 2 - 5 } \multicolumn{1}{c|}{} & \multicolumn{2}{c|}{ Center Jet } & \multicolumn{2}{c|}{ Ambient air } \\
\cline { 2 - 4 } \multicolumn{1}{c|}{} & $\mathrm{t}_{0}$ & $\mathrm{t}_{0}+\Delta \mathrm{t}$ & $\mathrm{t}_{0}$ & $\mathrm{t}_{0}+\Delta \mathrm{t}$ \\
\hline Run A & \multirow{2}{*}{7} & 5 & \multirow{2}{*}{4} & 3 \\
& & 4 & & 2 \\
\hline
\end{tabular}

Table 3: SNR for the PLIF images of Section III.A. 
Addressing the accuracy, precision, and overall uncertainty in the measurement was difficult in these preliminary measurements due to the low number of samples available for calculation. However, some assessment of the measurement precision was made based on both the measured variance in the velocity distribution as well as past works in the MTV literature. The measurement precision (based on one standard deviation of a series of repeated measurements) was found to vary considerably with both SNR and $\Delta t$. Near the core of the jet, where the SNR was highest, the precision, based on one standard deviation of the measurements, was approximately $0.8 \mathrm{~m} / \mathrm{s}$ for $\Delta t=100$ $\mu \mathrm{s}$ (Run A) and $0.6 \mathrm{~m} / \mathrm{s}$ for $\Delta t=200 \mu \mathrm{s}$ (Run B). Near the edges of the jet (ambient air), where the SNR was lower, a similar variation was observed: the precision for Run A was found to be $1.0 \mathrm{~m} / \mathrm{s}$ in these regions and Run B yielded $0.8 \mathrm{~m} / \mathrm{s}$, respectively. It was thus observed that precision worsened with decreasing SNR, while simultaneously being improved with longer time delays. The data of Bathel, et al. [12], obtained from a similar measurement system and technique, provides a reference against which to assess the trends in precision. They found the measurement precision to improve inversely with the time delay used. Extrapolating their results to the conditions of the current study, it can be estimated that the measurement precision for Run A should lie in the range 0.75 to $1 \mathrm{~m} / \mathrm{s}$ in the core of the jet, and in excess of $1.25 \mathrm{~m} / \mathrm{s}$ in the tails of the jet where the SNRs were substantially lower. They estimated the precision for Run B should lie the range of 0.45 to $0.7 \mathrm{~m} / \mathrm{s}$. In comparing these estimations to the current measured precision, the values are consistent, suggesting that the methodology used in measuring the velocities is similarly precise to other similar available methods.

It is important to note that these estimations of the measurement precision still represent a substantial fraction of, or in some cases exceed, the measured velocities, particularly in the ambient air region where the gas is nominally quiescent. Improving the measurement precision will require an increase in SNR and/or further increasing the temporal delay between images. These improvements were realized thereafter with a heated jet, and PLIF excitation in the $(1,0)$ to allow the use of spectral filters.

\section{B. HTV at $282 \mathrm{nm:}(\mathbf{1 , 0 )}$ transition}

The cases presented in this section are described in Tab. 2, which are thereafter referred by their Run letter. Figure 5 presents raw instantaneous PLIF images of Run C. The gas is air, similarly to Runs A and B, but at higher temperature to improve radicals production ( $373 \mathrm{~K}$ vs $298 \mathrm{~K}$ ), and longer $\Delta t$ for the second pulse ( $250 \mu \mathrm{s}$ vs 100 and $200 \mu \mathrm{s}$ ). Excitation at $282 \mathrm{~nm}$ and imaging at longer wavelength effectively removed scattered light that were visible in Fig. 3.

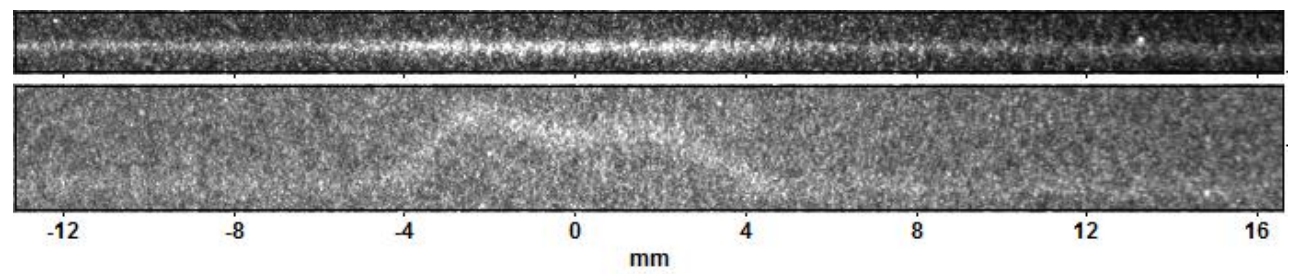

Figure 5: Raw instantaneous images of Run C. $t=t_{0}=10 \mu \mathrm{s}$ (top) $t=t_{0}+\Delta t=260 \mu \mathrm{s}$ (bottom)

The PLIF images of the radicals obtained with the first pulse, about $t_{0}=10 \mu \mathrm{s}$ after radicals formation, are averaged over 300 samples and shown in Fig. 6 for all four cases, with identical color scale [0-4000]. The first read pulse energy is $4 \mathrm{~mJ}$.

\section{$\mathrm{C}$}

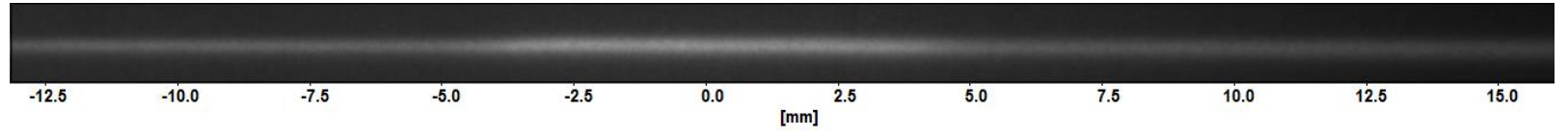

$\mathrm{D}$

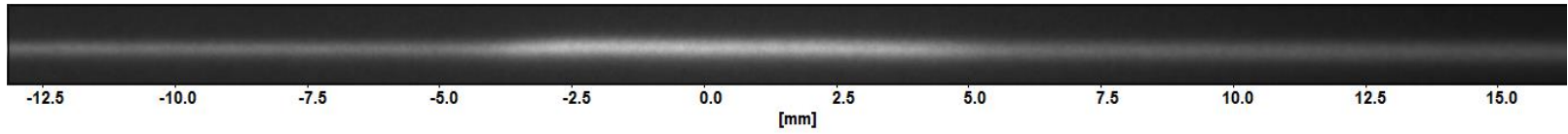

$\mathrm{E}$

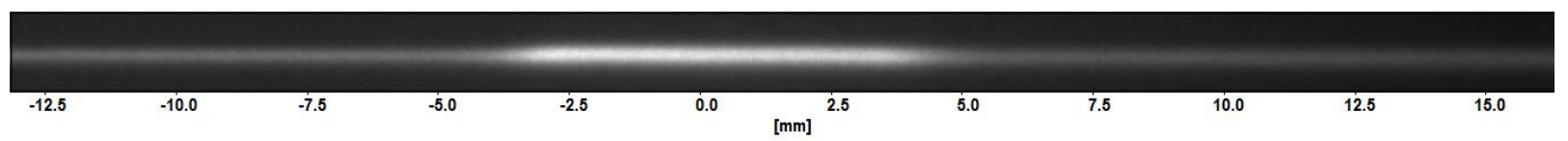


$\mathrm{F}$

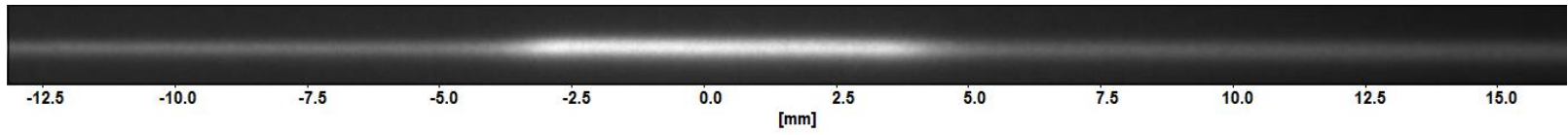

Figure 6: Averaged PLIF images of the first read pulse $10 \mu$ s after radical formation for Run C, D, E, and F.

The jet is located between $x=-5$ and $5 \mathrm{~mm}$. The higher vapor content there leads to a better signal compared to ambient air on both sides of the jet. The increase in signal between Run C and D confirms the benefits of increasing the temperature. The use of nitrogen in Run $\mathrm{E}$ and $\mathrm{F}$ also increases the signal compared to air in cases $\mathrm{C}$ and $\mathrm{D}$. The ambient air in which the jet discharges is identical in all 4 cases, thus the signal is similar is that region. The averaged PLIF images for the second read pulse are shown in Fig. 7 for all four cases. The color map is different from the previous figure, but constant within these four images [400-2000]. Second read pulse energy is 30mJ for all but Run E (20mJ).

$\mathrm{C}$

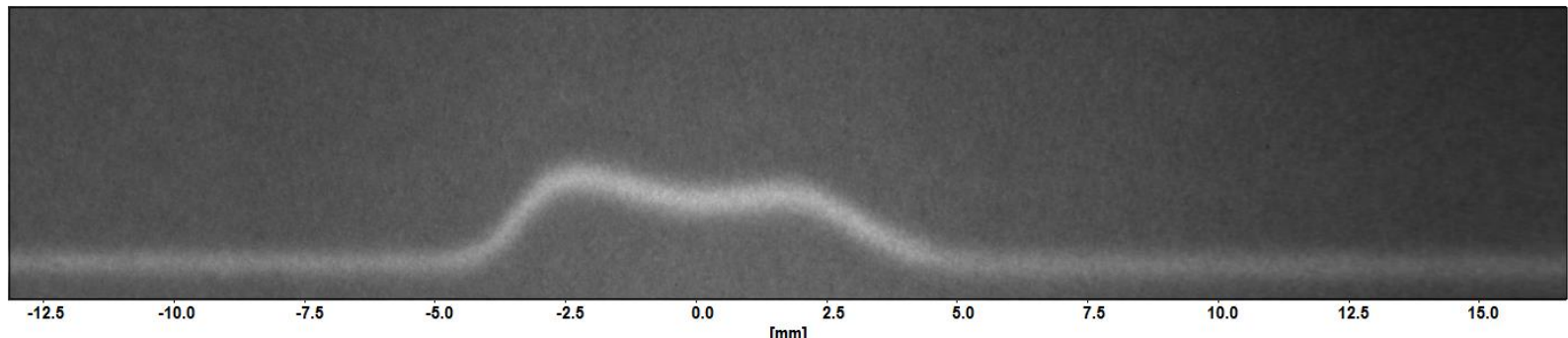

[mm]

D

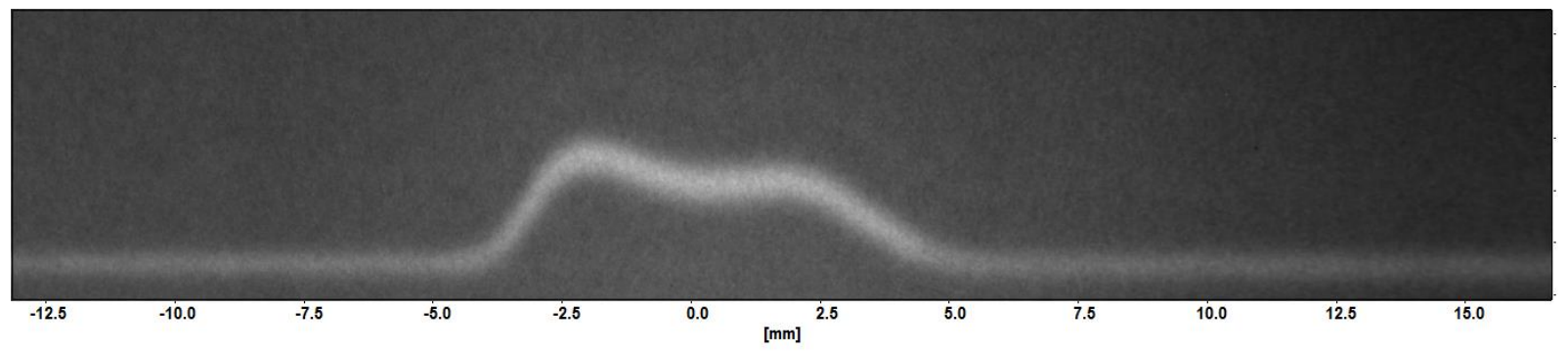

$\mathrm{E}$

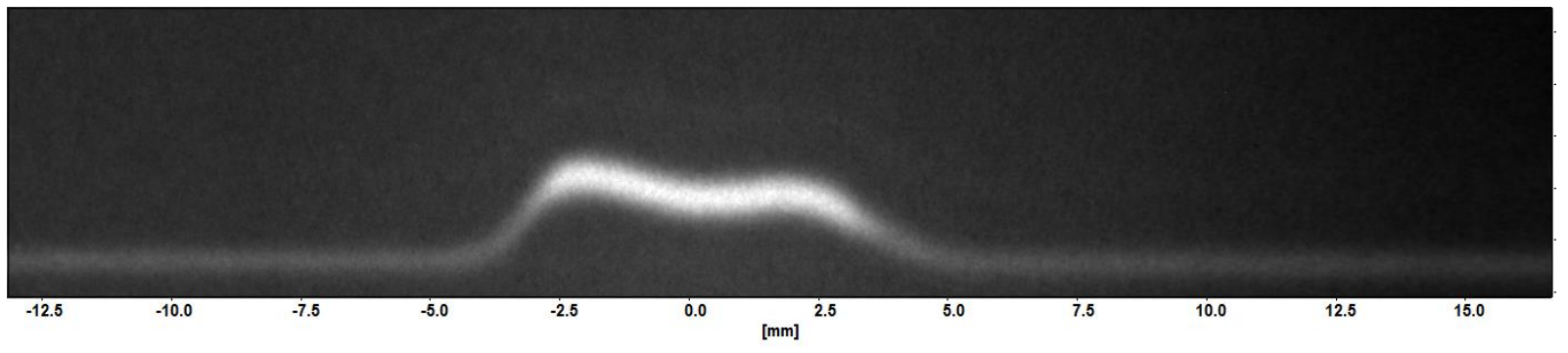

$\mathrm{F}$

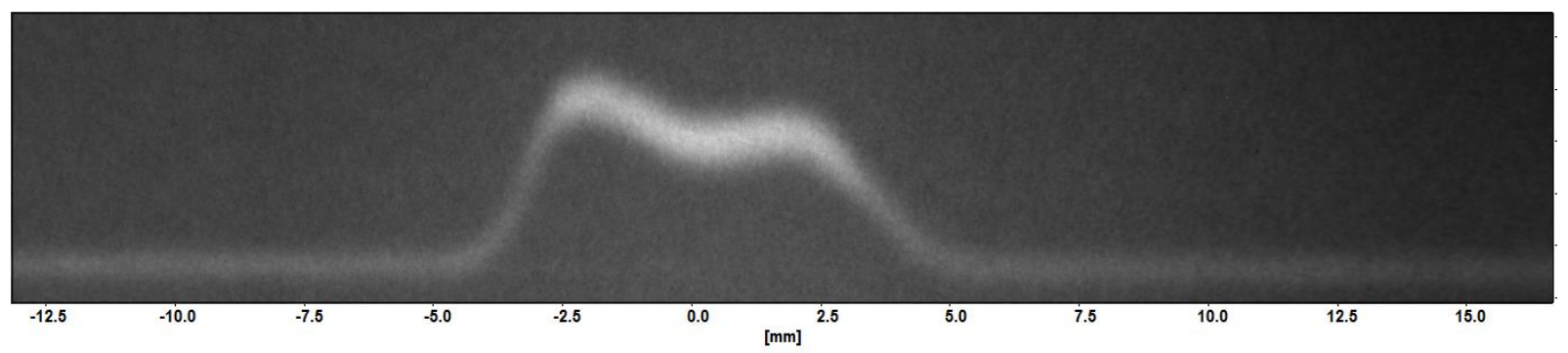

Figure 7: Averaged PLIF images of the second read pulse for Run C, D, E, and F.

Table 4 shows that in all cases, the SNR of the jet has markedly decreased compared to the first read pulse, but it is as good or better than for the $308 \mathrm{~nm}$ excitation of Section III.A (where $\Delta t$ was actually lower), showing the benefits of increasing temperature and filtering the fluorescent signal. OH absorption is lower at $282 \mathrm{~nm}$ than at 308 $\mathrm{nm}$, but the filtering of scattered light allows increasing the pulse energy. SNR in the ambient air is improved for the 
first read pulse due to the absence of scattering particles, but remains similar to previous data for the second read pulse. The displacement of the tagged line is much larger because of the increased $\Delta t$, which will result in a better precision. However, increasing the time delay has other consequences in terms of spatial and temporal resolution. The spatial resolution of MTV scales, to first order, with the recorded distance traveled by the tracer molecules. Hence, increasing the temporal delay improves the precision, but decreases the spatial and temporal resolution.

\begin{tabular}{|l|c|c|c|c|}
\cline { 2 - 5 } \multicolumn{1}{c|}{} & \multicolumn{2}{c|}{ Center Jet } & \multicolumn{2}{c|}{ Ambient air } \\
\cline { 2 - 5 } \multicolumn{1}{c|}{} & $\mathrm{t}_{0}$ & $\mathrm{t}_{0}+\Delta \mathrm{t}$ & $\mathrm{t}_{0}$ & $\mathrm{t}_{0}+\Delta \mathrm{t}$ \\
\hline Run C & 7 & 4 & 6 & 3 \\
\hline Run D & 14 & 4 & 7 & 3 \\
\hline Run E & 19 & 7 & 7 & 3 \\
\hline Run F & 19 & 5 & 8 & 3 \\
\hline
\end{tabular}

Table 4: SNR for instantaneous PLIF images of Section III.B.

The signal of Run D is still slightly better than Run C, but more diffuse because molecular diffusion increases with temperature. The increase of temperature also results in an increase in jet velocity (gas is fed a constant mass flow rate). The use of a non-reactive gas (Run E and Run F) clearly improves the signal, which allows maintaining a sufficient SNR for $\Delta t$ of at least $500 \mu$ s (Run F). Note that in Run E the second read pulse energy was lower than in the 3 other cases $(\sim 20 \mathrm{~mJ}$ vs $\sim 30 \mathrm{~mJ})$. It was noticed that the fluorescence intensity saturates for read pulses above 15 $\mathrm{mJ} / \mathrm{pulse}$ for the present setup, therefore any increase in read pulse energy only increases the background noise without improvement in the signal, and thus decreases the SNR. This is an important point which shows that the probe pulse energy must be tuned to maximize SNR instead of just the signal.

About 300 image pairs are recorded for each Run. Each image pair is processed independently, with a horizontal binning of only 20 pixels this time. The mean velocity profiles and RMS fluctuations are shown in Fig 8 .

(a)

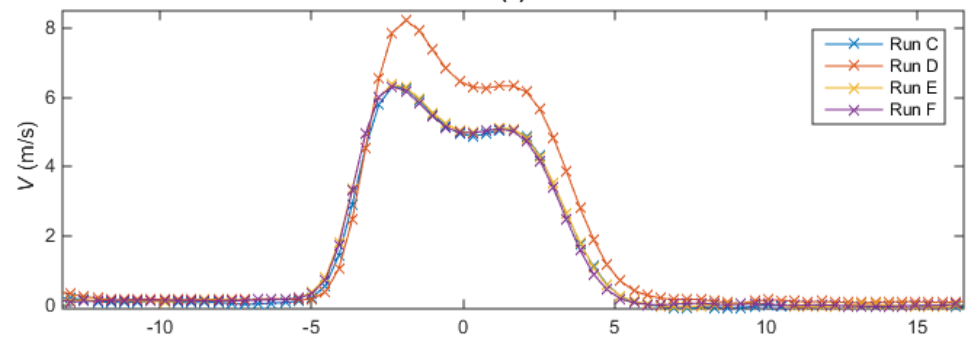

(b)

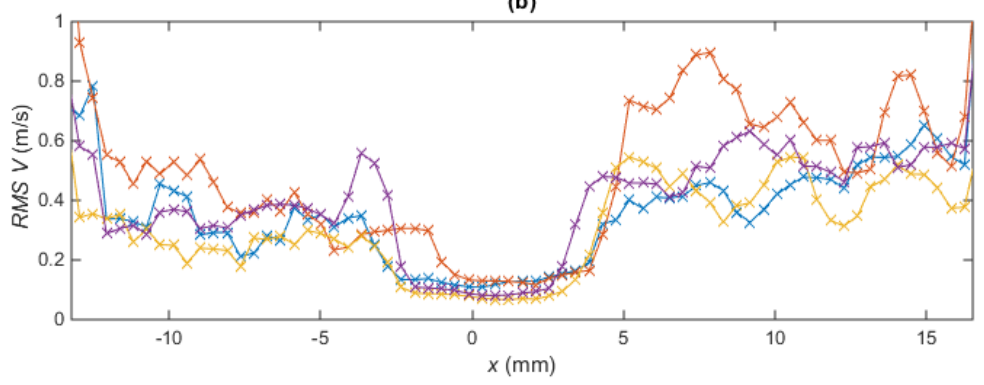

Figure 8: (a) Mean velocity profiles. (b) RMS of velocity fluctuations.

Run D stands out as its velocity differs from the 3 other cases because of the higher temperature. Using the ideal gas law, the increase factor in mean velocity is estimated at $T_{2} / T_{1}=473 \mathrm{~K} / 373 \mathrm{~K}=1.27$. The measured increase ratio is between 1.25 and 1.34 for the $-2 \mathrm{~mm}<x<2 \mathrm{~mm}$ region, which agrees with the $\mathrm{T}_{2} / \mathrm{T}_{1}$ ratio, thereby explaining the discrepancy. The RMS fluctuations for Run D are also higher possibly because fumes coming from the heat tape interfered with the signal. For these reasons, discussion of Run D will be limited in the following.

The mean profiles of the other three cases are very similar with a maximum velocity of $V_{\max }=6.5 \mathrm{~m} / \mathrm{s}$. This was expected since the pressure and temperature of the gas was kept the same. The velocity profile is asymmetrical, which is not surprising since no flow conditioning was used. For this steady flow, the RMS fluctuations give the 
precision of the measurement. Near the center of the jet, where the SNR is the highest, the precision is $0.12 \mathrm{~m} / \mathrm{s}$ $\left(1.8 \% V_{\max }\right), 0.08 \mathrm{~m} / \mathrm{s}\left(1.2 \% V_{\max }\right)$, and $0.10 \mathrm{~m} / \mathrm{s}\left(1.5 \% V_{\max }\right)$, and for Runs C, E, and F, respectively. This is a significant improvement over the results of Section III.A. In the regions away from the jet, the mean velocity is measured between -0.1 and $0.2 \mathrm{~m} / \mathrm{s}$. The precision is similar for all three cases since the gas is identical there (air). It is between $0.3 \mathrm{~m} / \mathrm{s}$ and $0.6 \mathrm{~m} / \mathrm{s}$. This is higher than in the jet because of the low SNR in ambient air, but still an improvement over the unfiltered case of Section III.A.

\section{Comparison with PIV and uncertainty estimation}

HTV measurements are now compared with these obtained with PIV in the same flow conditions as Run C (air at $100{ }^{\circ} \mathrm{C}$ and $\Delta t=250 \mu \mathrm{s}$ ) using the same instruments and almost identical setup. Such a configuration allows a fair comparison between the two methods, since the camera imaging and calibration, investigation plane (dye laser sheet dimensions and location), and pulse timing are virtually identical. To perform PIV, the long pass filters are removed from the camera, and the excimer laser is not fired. Instead, water droplets tracers are generated by the atomizer in addition of the constant flow that is bubbled. To keep the flow rate identical between PIV and HTV, droplet generation is stopped just before the recording starts. Droplets remaining in the atomizer reservoir then seed the flow. This limits the duration of each run to about 6 seconds, or 60 image pairs. Therefore, several runs are recorded to get a similar number of samples as in each HTV run ( $\sim 300$ vector fields). Continuous seeding would also be possible by diverting some of the bubbling flow to the atomizing nozzle. Image pairs are cross-correlated using the software Davis 8.0.8 from LaVision, Inc, and mean and RMS vector fields are extracted.

PIV results for the mean velocity profile are shown in Fig. 9 for the downstream region corresponding to where HTV is performed, and compared to the results obtained with HTV. Because only the fluid issued from the nozzle is seeded, the entrained flow and ambient air velocity cannot be resolved with here, which results in erroneous measurements near the boundaries of the PIV domain. The velocity profiles are in good agreement in the jet, where the PIV images are adequately seeded. The observed difference in mean velocity between PIV and HTV in the center region is less than $2 \%$, and the RMS difference was found to be $1.2 \%, 0.8 \%$, and $1.0 \%$, for Run $\mathrm{C}$, E and F, respectively. Comparison cannot be made in the quiescent region of the flow, or for Run D since the jet velocity is different. PIV precision is measured at $0.04 \mathrm{~m} / \mathrm{s}\left(0.6 \% V_{\max }\right)$ on average in the center jet region, which is half of the RMS of the best HTV case, Run E.

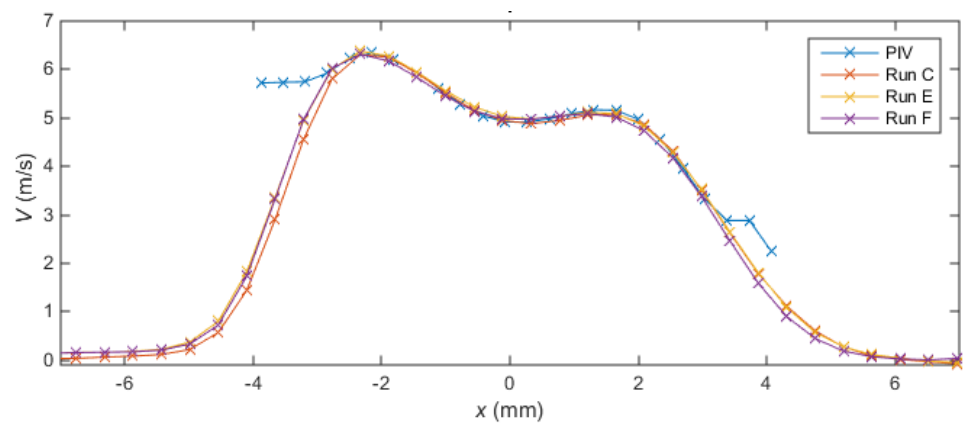

Figure 9: Mean velocity profile comparison between PIV and HTV

The agreement between PIV and HTV is very good - a consequence of using the same setup (camera, laser sheet, calibration) for both measurements. This experimental arrangement cancels out any systematic errors that could arise from these components, and shows that for a given setup, HTV is almost as good as PIV for resolving mean velocity. The accuracy of each measurement is calculated based on estimation of the error on calibration $(0.5 \%)$, timing (negligible), and displacement measurement. For the latter, an accepted guideline is 0.2 pixel for PIV [2]. For HTV, a conservative choice is to use the RMS difference between PIV and HTV by assuming PIV is more accurate than HTV. Accuracy and precision are combined to calculate the measurement uncertainty, which is presented at $68 \%$ confidence level in Tab. 5 .

The best uncertainty is obtained for Run E, which combines the advantages of hot and inert gas, with moderate $\Delta t$. The increased of $\Delta t$ in Run F leads to a lower SNR, which decreases accuracy, but would otherwise be beneficial for studying slower flows to allow for a larger displacement and thus improve precision. Measurements in heated air jet also show decent uncertainties. Results for the ambient air are slightly better than for the HTV results in the $(0,0)$ 
band, but the error is still considerable. Absolute error on the order of that in the center jet can be expected for a quiescent gas in the same condition and composition as the jet.

\begin{tabular}{|l|c|c|}
\hline & Center jet & Ambient air \\
\hline PIV (Air $\left.100^{\circ} \mathrm{C} \Delta t=250 \mu \mathrm{s}\right)$ & $0.06 \mathrm{~m} / \mathrm{s}(0.85 \%)$ & Not resolved \\
\hline Run $\mathrm{C}\left(\right.$ Air $\left.100^{\circ} \mathrm{C} \Delta t=250 \mu \mathrm{s}\right)$ & $0.14 \mathrm{~m} / \mathrm{s}(2.2 \%)$ & $0.34 \mathrm{~m} / \mathrm{s}$ \\
\hline Run D (Air $\left.200^{\circ} \mathrm{C} \Delta t=250 \mu \mathrm{s}\right)$ & $0.19 \mathrm{~m} / \mathrm{s}(2.3 \%)$ & $0.40 \mathrm{~m} / \mathrm{s}$ \\
\hline Run E $\left(\mathrm{N}_{2} 100^{\circ} \mathrm{C} \Delta t=250 \mu \mathrm{s}\right)$ & $0.10 \mathrm{~m} / \mathrm{s}(1.5 \%)$ & $0.27 \mathrm{~m} / \mathrm{s}$ \\
\hline Run $\mathrm{F}\left(\mathrm{N}_{2} 100^{\circ} \mathrm{C} \Delta t=500 \mu \mathrm{s}\right)$ & $0.12 \mathrm{~m} / \mathrm{s}(1.9 \%)$ & $0.40 \mathrm{~m} / \mathrm{s}$ \\
\hline
\end{tabular}

Table 5: Single shot velocity uncertainties at $68 \%$ confidence level

\section{Conclusion}

The present study demonstrates the possibility to conduct measurements using the HTV technique in low-speed (few $\mathrm{m} / \mathrm{s}$ ) and ambient- or modestly-elevated-temperature flows. While PIV is traditionally suited for such flows, HTV can be favored when the experiment presents constraints for PIV such as extended period of quiescent flow, where particles may settle, or in applications where the introduction of particles is not possible. The applicability of the present test flow to both PIV and HTV allows a direct comparison of experimental results obtained with each technique.

This study finds that the best HTV results are obtained using (1,0) transition around $282 \mathrm{~nm}$, allowing use of a spectral filter to reject noise at the laser's wavelength, improving the SNR. This is not possible for resonant fluorescence in the $(0,0)$ band, although Section III.A shows that the flow can still be resolved, albeit with higher uncertainties. The effect of gas temperature, composition, and $\Delta t$ on SNR and uncertainty have been analyzed. HTV results agree well with PIV, and precision of the two methods can be compared. Although HTV error is still higher than PIV error (best case scenario $1.5 \%$ vs $0.85 \%$ ), such a value is sufficient in many velocimetry applications.

These results show that the HTV technique, put in context with past work, can readily be implemented with good accuracy over a very wide range of velocity $(<1 \mathrm{~m} / \mathrm{s}$ to $>$ Mach 2$)$ and temperature $(300 \mathrm{~K}$ to $>2000 \mathrm{~K})$ with virtually no change in the experimental setup except the value of $\Delta t$. Directly comparing to past work, single shot measurement uncertainties as low as $0.10 \mathrm{~m} / \mathrm{s}$ (68\% confidence level) were obtained, which is an order of magnitude lower uncertainty than the prior best single shot uncertainty described in the literature, which is summarized in Tab. 1.

Finally, the use of a pair of probe lasers was used for HTV for the first time, defending against errors that could be caused by misalignments of the excitation and probe beams.

\section{Acknowledgments}

This project was supported by a DOE NEUP grant to Dr. Bardet.

\section{References}

1. Springer Handbook of Experimental Fluid Mechanics, ed. Topea, Yaris, Foss, ch. B.5.4.

2. Adrian, R. J., "Twenty Years of Particle Image Velocimetry," Experiments in Fluids, Vol. 39, 2005, pp. 159-169.

3. Danehy, Paul M., et al. "Flow-tagging velocimetry for hypersonic flows using fluorescence of nitric oxide." AIAA journal 41.2 (2003): 263-271.

4. Andre, M. A., et al. "Molecular Tagging Velocimetry development for in-situ measurement in High-Temperature Test Facility" Proceedings of the 2015 ANS winter meeting, Washington, DC

5. Pitz, Robert W., et al. "Unseeded molecular flow tagging in cold and hot flows using ozone and hydroxyl tagging velocimetry." Measurement Science and technology 11.9 (2000): 1259.

6. Ribarov, L. A., et al. "Hydroxyl tagging velocimetry (HTV) in experimental air flows." Applied Physics B 74.2 (2002): $175-183$

7. Ribarov, L. A., et al. "Multiline hydroxyl tagging velocimetry measurements in reacting and nonreacting experimental flows." Experiments in Fluids 37.1 (2004): 65-74.

8. J. Luque and D.R. Crosley, "LIFBASE: Database and Spectral Simulation Program (Version 1.5) ", SRI International Report MP 99-009 (1999). 
9. Pitz, Robert W., et al. "Hydroxyl tagging velocimetry in a supersonic flow over a cavity." Applied optics 44.31 (2005): 6692-6700.

10. Wehrmeyer, Joseph A., et al. "Flame flow tagging velocimetry with 193-nm $\mathrm{H}_{2} \mathrm{O}$ photodissociation." Applied Optics 38.33 (1999): 6912-6917.

11. Lahr, M. D., et al. "Hydroxyl-tagging-velocimetry measurements of a supersonic flow over a cavity." Journal of Propulsion and Power 26.4 (2010): 790-797.

12. B. Bathel, P. M. Danehy, C. Johansen, S. Jones, C. Goyne, "Hypersonic Boundary Layer Measurements with Variable Blowing Rates Using Molecular Tagging Velocimetry," Paper AIAA-2012-2886, 28th Aerodynamic Measurement Technology, Ground Testing, and Flight Testing Conference, New Orleans, Louisiana, June 25-28, 2012 